\title{
Guidelines for Writing Letters to Patients
}

\author{
Diane L. Baker, ${ }^{1}$ Timothy Eash, ${ }^{2}$ Jane L. Schuette, ${ }^{1,3}$ \\ and Wendy R. Uhlmann ${ }^{1,4,5}$
}

\begin{abstract}
Patient letters provide a permanent record of the genetic counseling that was provided and are unique in medical care; rarely do other health care providers send summaries written specifically to their patients and families. We surveyed genetic counseling training program directors and found that while the acquisition of patient letter-writing skills was considered important, there were no specific guidelines made available to students. To develop letter-writing guidelines, we evaluated patient letters, reviewed references on professional correspondence, surveyed the medical literature, and worked with a writing consultant. The guidelines we subsequently developed and present here include a format for writing patient letters, suggestions on presenting medical information in understandable terms, and wording considerations. These patient letter-writing guidelines are intended to serve as a guide for teaching students this important skill and as a resource for practicing health care professionals.
\end{abstract}

KEY WORDS: patient letters; genetic counseling; genetic counselors; people-first language; active voice; passive voice; confidentiality; communicating distance.

\footnotetext{
${ }^{1}$ Department of Human Genetics, University of Michigan, Ann Arbor, Michigan.

${ }^{2}$ Writing Consultant, New York, New York.

${ }^{3}$ Division of Pediatric Genetics, Department of Pediatrics, University of Michigan, Ann Arbor, Michigan.

${ }^{4}$ Division of Medical Genetics, Department of Internal Medicine, University of Michigan, Ann Arbor, Michigan.

${ }^{5}$ Correspondence should be directed to Wendy R. Uhlmann, MS, CGC, Division of Medical Genetics, University of Michigan, 4301 MSRB III, Box 0638, Ann Arbor, Michigan 48109-0638; e-mail: wuhlmann@umich.edu.
} 


\section{INTRODUCTION}

The patient letter has long been recognized as a valuable and vital tool of the genetic counseling process (Kelly, 1977). It serves as a permanent summary of the genetic counseling that was provided and documents pertinent aspects of the patient's medical history, family history, and genetic circumstances. The letter also supports the patient's ongoing self-understanding and provides a mechanism by which information about their genetic condition can be shared with others. The usefulness of patient letters was documented in Hallowell and Murton's study of women seen for genetic counseling for breast/ovarian cancer (Hallowell and Murton, 1998). Ninety-two percent of study participants indicated that the patient letter facilitated their understanding and/or recall of information and $85 \%$ indicated that they had shared or intended to share their letter with relatives (Hallowell and Murton, 1998).

While patient letters are an important resource for patients, family members, and caregivers, there have been no established guidelines for content, format, or wording of these letters. Many genetic counselors (including the authors) have developed letter-writing skills by trial and error over time. As genetic counseling training program faculty, we found that our efforts to assist students in acquiring this competency were limited to providing students with sample letters early in their training and then critiquing their draft letters during clinical rotations. Although their letter-writing skills evolved, we found that students continued to encounter difficulties in organizing the content of the letter, minimizing the use of complex language when discussing genetic and medical concepts, describing imprecise patient circumstances and avoiding wordiness.

We hypothesized that letter-writing skills could be taught more effectively if the techniques used by practicing genetic counselors were more systematically examined, enumerated, and illustrated. We therefore initiated a process that would allow us to identify and document the methods by which genetic counselors throughout North America compose individualized patient letters. We report here, in the form of guidelines, the outcome of these efforts. These letterwriting guidelines are specific to the general genetics clinic; however, their format is readily adaptable to other types of clinic visits and counseling situations. The writing of physician letters and chart notes are not addressed in these guidelines.

\section{METHODS}

We identified a writing consultant (TE) to collaborate with us in analyzing patient letters from the pediatric and adult genetics clinics at our center. This analysis identified elements of style, language usage, content, and construction 
common to these letters. We reviewed the literature on patient correspondence and designed a survey for genetic counseling programs about their methods of instruction students in letter writing. In April 1995, we sent the survey to 19 genetic counseling program directors. Responses received from 14 (74\%) program directors indicated that the acquisition of patient letter-writing skills was considered important yet there were no standard guidelines for teaching these skills. Working with the writing consultant, we drafted a preliminary document that defined some basic elements of patient letter writing culled from our internal analysis and the national survey. This draft document was sent to 19 program directors, 40 clinical supervisors, and 67 recent graduates for review in April 1996. Feedback from these reviewers was incorporated and in February 1997, a revised set of guidelines was sent to program directors and clinical supervisors for use in teaching letter writing to their students. A follow-up survey was sent in May 1997 to this same group of program directors and supervisors to learn about their experience piloting the guidelines.

The results of these efforts and surveys are the patient letter-writing guidelines that appear below. The guidelines themselves are more of an analysis of the components of a patient letter, rather than a step-by-step instructional guide. The guidelines are divided into two sections, the basics of writing for patients (Part I) and what we call additional considerations (Part II). Part I reviews basics writing skills and provides suggestions on how counselors may deal with medical and genetic information in the letter. Part II addresses the somewhat more elusive aspects of writing for patients such as the use of distance, active versus passive voice, point of view, people-first language, and the presentation of patient affect. Although defining the elements of the genetic counseling patient letter can help students become more conscious of the subtleties of letter writing, it is important to keep in mind that not all elements described here will be included in every letter. In addition, writing styles will vary from counselor to counselor and from letter to letter.

\section{PATIENT LETTER-WRITING GUIDELINES}

\section{Purpose and Format of the Patient Letter}

The patient letter is written directly to the patient or the parent/guardian of a patient for the purpose of providing a permanent, easily understood record of the relevant information discussed during the genetics clinic visit. The patient letter also addresses information that may have become available since the patient's visit (e.g., outside records or laboratory results). The letter is a carefully written summary of the patient's visit and it reinforces the relationship between the patient's own circumstances and specific genetic and medical information. It is generally 
composed on behalf of the entire genetics term, including the geneticist, genetic counselor, and others who were involved in the clinic visit. The patient letter serves two functions:

1. To record relevant facts

- The letter summarizes pertinent medical and family history information; reviews the medical findings of the visit; documents risk figures, recommendations and referrals; and addresses questions raised by the patient during the visit.

- The letter is part of the patient's permanent medical record and therefore genetic counselors will want to be familiar with their sponsoring institution's guidelines on medical and legal accountability regarding written documents.

2. To present information in a manner that promotes the patient's understanding

- The letter is for the patient's understanding and reference and should be written in clear and concise language. To facilitate this goal, the finished letter should be reviewed from the perspective of the patient regarding the following: Is it clear? Is it accurate? Is it nonstigmatizing?

- A secondary audience for the letter, should the patient wish to share it, may include other health care providers, family members, teachers, school administrators, or child care workers.

Patient letters should be concise and generally are no longer than two pages in length. Just as information in a counseling session is presented in a manner that promotes patient understanding by building on explanations of basic genetic concepts, so does the patient letter. The letter template shown in Table I outlines the typical components of a patient letter. The order of the various sections is flexible and is supersended by any standardized formats used in a particular center. Furthermore, the nature of the genetic counseling interaction (e.g., presymptomatic testing vs. informing about a specific diagnosis) may necessitate a different ordering of the information.

\section{Privacy and Confidentiality}

Patient letters are generally included in hospital charts and clinic records; therefore, the same professional and institutional standards of privacy and confidentiality that apply to medical records apply to the patient letter. The reader is referred to the National Society of Genetic Counselors Code of Ethics (National Society of Genetic Counselors, 1992) and to your own institution's guidelines for clarification about the privacy and confidentiality of medical records. In addition, 
Table I. Sample Template for Patient Letters

Genetics Clinic Letterhead

Date

Family address

Re: Patient name

Dear (Patient, Parent, or Guardian):

Hospital registration number

I. Introduction (generally only a few sentences in length)

Date of visit

Clinic identification

Reason for referral or visit (include name of referring provider)

Purpose of the letter

II. Body

A. Description of significant family, medical, pregnancy, and developmental histories and pertinent test results ${ }^{a}$

B. Review of physical examination and/or diagnosis ${ }^{a}$

Findings on physical exam/basis for diagnosis

Interpretation of significant $(+)$ or $(-)$ family history

C. Natural history of condition

Description of clinical features and prognosis

Incidence of condition and carrier frequency in general population

D. Explanation of inheritance

Brief description of chromosomes and genes, if relevant

Suspected or established inheritance pattern(s)

E. Summary of risk assessment

Recurrence risks

Risks to other family members

Baseline risk of birth defects, if relevant

F. Recommendations for medical management, diagnostic work-up, genetic testing, referrals, ${ }^{b}$ etc.

Clear, unambiguous summary of recommendations

Laboratory testing undertaken or under consideration ${ }^{b}$

G. Outline of reproductive options/prenatal diagnosis

$\mathrm{H}$. Significant psychosocial issues and concerns

I. Recommendations of nonmedical resources (including contact names, telephone numbers, etc.)

Support groups, support services

Patient literature

Educational resources

III. Closing

Plan for reporting test and/or evaluation results

Date of return visit to genetics clinic

Invitation to recontact genetics clinic

Other closing remarks

IV. Signatures

Genetic counseling student

Staff genetic counselor

Fellow

Attending geneticist

cc: Local physician/others as requested by family

${ }^{a}$ Reference the source of this information (i.e., patient/parent report, physical examination, consultant report, medical institution/laboratory from which outside records were obtained, etc.).

${ }^{b}$ This information should be referenced (e.g., to which clinic a referral was sent, to which lab a specimen was sent, etc.). 
the genetic counseling process routinely records family names, biological relationships, and other identifying information about family members. When referring to family members in the counseling letter, care needs to be taken to ensure that their privacy is protected when documenting their medical and genetic information. Since letters are often shared with other family members, it is important to discuss with the patient what family information will appear in the letter.

If the patient is an older teen, determine to whom the letter will be addressedthe parent, the child-patient, or a separate copy to each. This determination should be addressed as part of the counseling session and decided on a case-by-case basis. If patients request that you send copies of their letter to providers, educators, or other, discuss whether the complete or an abbreviated version of the letter should be sent.

\section{The Process of Writing Patient Letters}

This process begins by determining the information to document in the letter based on what transpired during and subsequent to the patient visit. Letters are not meant to be a transcript of the entire clinic visit, but a clear and succinct summary of the pertinent information. It is important to take into consideration both the patient's level of education and cultural background when wording the letter. Some genetic terms (e.g., chromosome, gene, etc.) and concepts (e.g., Mendelian patterns of inheritance, prenatal testing, etc.) are frequently utilized in patient letters, and therefore, it is helpful to develop a stockpile of standardized descriptions to use in letters. While having standardized text available can save time, it is important to not simply drop text into a letter but to modify this information so that it appropriately fits each patient.

\section{PART I: THE WRITING BASICS FOR PATIENT LETTERS}

This section presents general writing guidelines that help define and clarify the letter-writing process. While this information will be quite familiar to many, it is attentiveness to these basic skills that can make the difference between a well-crafted letter and one that does not serve the patient's needs. We wish to emphasize that the following are general writing recommendations and there will be many situations in which a genetic counselor may choose to structure or present information differently from these guidelines.

\section{Clear, Simple Writing}

Clear, easily understood writing is the most important skill needed for writing patient letters. In general the counselor seeks to relay information in a 
straightforward and unambiguous style, avoiding abstractions, complex terminology, or wordy explanations. The patient's level of comprehension of medical and genetic concepts should be considered and accommodated, so that the counselor avoids medical jargon and information gaps that only a medical professional would understand. Sentences should be kept short and simple, but not choppy, and should usually contain only one idea (Blake and Bly, 1991; Venolia, 1982). However, there may be circumstances in which it is necessary to use longer, more descriptive statements. Transitional words and phrases between sentences and ideas should be used to help guide the reader along (Venolia, 1982).

\section{Paragraph Construction}

The best structural device available for developing an effective patient letter is the paragraph. The paragraph allows complex information to be broken down into more understandable sections (Blake and Bly, 1991; Strunk and White, 1979; Venolia, 1982). Ideas should flow in a logical manner from one sentence to another and from one paragraph to the next, resulting in a document that is readable and complete. From beginning to end, the letter should, simply put, make sense.

The conventional paragraph begins with an introduction, a sentence or two that introduce clearly to the reader what will be contained in the paragraph. This is followed by the body of the paragraph, in which the essential information on that topic is unfolded for the patient. The paragraph generally ends after the pertinent information on the specific topic at hand has been presented (Strunk and White, 1979).

Sample introductory sentences:

As you may recall, we discussed the implications of John's diagnosis for your current pregnancy. (Proceed to discuss the implications.)

In discussing the inheritance of Marfan syndrome, we began by reviewing dominant inheritance. (Proceed to discuss autosomal dominant inheritance.)

\section{Accurate Presentation of Medical and Genetic Information}

Medical and genetic information should be presented in the letter as explicitly as possible. Statements that do not withstand reasonable standards of accuracy or that cannot be substantiated should be avoided. All of the following examples in the left-hand column may be statements about which the counselor feels fairly certain; nonetheless, they are either statements about which the counselor cannot be absolutely certain or statements that imply a sense of confidence that may be unfounded. 
Less accurate

The blood test will determine if a chromosomal rearrangement is present.

There is nothing you did during your pregnancy that contributed to your child's condition.

By the time your daughter is of reproductive age, the gene ${ }^{b}$ for $\mathrm{XYZ}$ syndrome will probably have been identified.

\section{More accurate}

The blood test will determine if an identifiable chromosomal rearrangement is present. ${ }^{a}$

Based on the information available, there is nothing you did or did not do during your pregnancy that would have contributed to your child's condition.

Research on the gene responsible for $\mathrm{XYZ}$ syndrome is progressing, and we recommend that when your daughter is of reproductive age she seek updated genetic counseling for this condition.

\footnotetext{
${ }^{a}$ This example uses more complex language and may not be appropriate for all circumstances. ${ }^{b}$ While Mendelian inheritance results from the transmission of alleles, haplotypes, or genotypes, many genetic counselors for simplicity refer to transmission of a "gene" when communicating with patients (e.g., the cystic fibrosis or Tay-Sachs "gene").
}

\section{Explanation of Medical Terminology}

While some diagnostic and medical terminology needs to be included in a patient letter, its use should be limited to what is essential. Technical terms should be defined in language that the patient can easily understand and medical jargon should be avoided. For example, although the following phrases would be appropriate for a chart note, they should generally be avoided in the patient letter: "she tests positive/negative for ..., family history is unremarkable for ..., denies history of ...." When using acronyms, introduce the acronym parenthetically the first time and then use it consistently throughout the remainder of the letter. For example, "Alpha-fetoprotein (AFP) is ...." The following examples illustrate how genetic terminology can be reworded to promote patient understanding.

\section{Technical}

Down syndrome results from nondisjunction $^{a}$ of chromosome \#21.

The gene that causes this condition is on one of the autosomes. ${ }^{a}$

${ }^{a}$ Unless explained earlier in the letter.

\section{Simplified}

Down syndrome results from the presence of an extra chromosome \#21. The gene that causes this condition is on one of the \#4 chromosomes.

Parenthetically set off lay phrases can be used to define medical terms that are introduced. Some counselors also employ the use of italics or bold to set off important concepts or data within the letter. 
Examples:

During the examination Dana was noted to have hypotelorism (closely set eyes). Douglas exhibited several symptoms of neurofibromatosis, including Lisch nodules (areas of pigment on the iris of the eye).

\section{PART II: ADDITIONAL CONSIDERATIONS FOR PATIENT LETTERS}

This section addresses areas of letter writing that are subtler, more stylistic, and perhaps less universal than the preceding section. We have found that these techniques are more elusive and challenging for students to master and, therefore, this is a more extensive section than Part I. The appendix provides a sample letter and commentary about the use of the tools discussed below.

\section{Consideration of Distance and the Tools Used to Portray It}

When writing patient letters, counselors have the ability to choose how they would like to present information. For example, the letter will portray how definitive the staff feels about a particular diagnosis, how directly they wish to present information, and how formally or informally they wish to convey their relationship with a patient. These characteristics can be grouped under the concept of distance in regard to three different relationships.

1. Relationship between staff and patient.

2. Relationship between the staff and the information presented in the letter.

3. Relationship between the patient and the information presented in the letter.

The amount of distance conveyed in letters regarding any of these relationships will vary between different counselors, as a result of their individual styles, and from letter to letter for the same counselor in response to the unique nature of each case.

Tools that can be used to influence the sense of distance that is conveyed in a letter include active versus passive voice, point of view, and the use of definitive or nondefinitive statements.

\section{Distance: Relationship Between Staff and Patient}

While the style and tone of the entire letter convey how the staff/patient relationship is viewed, the amount of distance between staff and patient is usually more obviously revealed in the letter's introductory and closing remarks and when addressing the patient's specific situation (family history, risks, and examinations). Sometimes it may seem appropriate to hold a more distant or formal relationship with a patient, while at other times it may seem appropriate to minimize the distance 
in that relationship. The following examples show how varying degrees of distance between the staff and the patient might be conveyed.

\section{Closer}

We are sorry that your partner was unable to attend the visit and hope this letter will serve to review for him the information that was discussed.

It was a pleasure to meet both of you and your two young sons, Daniel and David.

We were so sad to learn of your recent miscarriage. Over this past year we have come to understand how much you looked forward to having another child.

\section{More distant}

This letter will also serve to review for your partner the significant information discussed.

This letter summarizes the information discussed regarding both of your children.

We want to extend our condolences on the recent the loss of your pregnancy.

One way to reduce distance and communicate the staff's genuine interest in the patient is to include a few personal details, which signify that they were seen and heard as a complete person and not just as a manifestation of a specific genetic condition.

Examples:

It is clear that Sam's spina bifida has not had any effect on his singing abilities, and we certainly enjoyed his demonstration.

Because of your planned wedding next May, perhaps you would like to schedule your return visit in April or June.

\section{Distance: Relationship Between Staff and Information}

Health care professionals need to consider how definitive they are willing to be about diagnoses and recommendations for treatment, given issues of uncertainty and evolving technology. In certain circumstances (ambiguous clinical findings, uncertain diagnoses, etc.), the genetic counselor may choose to present information in a manner that distances the clinic staff from the information. This can be accomplished by using qualifying and modifying statements, as illustrated in the following examples:

\section{Closer}

We noted bumps on your back, which are consistent with neurofibromas.

We made the diagnosis of ...

\section{More distant}

Physical examination of your back revealed bumps, which exhibit characteristics of neurofibromas.

The results of the examination performed were consistent with the diagnosis of... 
Likewise, the counselor and other members of the clinic staff can be left out of certain passages. This exclusion of the staff will tend to increase the distance from the information conveyed. There are circumstances when this may be appropriate, as in situations of ambiguous information, discussion of new but unconfirmed findings, or in describing a circumstance that requires further investigation. It is also true that exclusion of the staff may de-emphasize the importance of the information in the letter. Again, it is the counselor's judgment that will guide the use of this technique.

\section{Including staff}

We observed dramatic improvement of Karen's physical abilities.

Dr. Chow diagnosed neurofibromatosis in your son.

\section{Excluding staff}

Dramatic improvement of Karen's physical abilities was observed.

Your son's features were felt to be consistent with the diagnosis of neurofibromatosis.

\section{Distance: Relationship Between Patient and Information}

The relationship between the patient and specific information is conveyed throughout most of the body of the letter, especially when citing risk information, diagnostic information, and follow-up plans. Because information disclosed during a genetics clinic visit can have very personal significance for patients, counselors may choose to modulate its impact by making powerful news seem, at least initially, further removed from the patient. Using rhetorical devices, the counselor can write in ways that seem less threatening and less direct-in other words, more distant from the patient. Notice the difference between two ways of saying basically the same thing:

\section{Closer}

Your test results indicate that you have inherited the gene alteration for Huntington disease.

You were diagnosed with Marfan syndrome.

\section{More distant}

According to the report received from the DNA Laboratory, the results of the analysis were consistent with having the alteration associated with Huntington disease.

Based on the physical features that were noted during the examination, you were diagnosed with Marfan syndrome.

While the examples on the right are wordier, they are also more distant from the reader than the examples on the left. Through the rearrangement and addition of words, the patient is given more time and space to identify with the information. Writing in this way allows the patient to realize that his circumstances or symptoms 
are connected with the diagnosis, without immediately connecting himself with the diagnosis. However, later on in the letter, it may be appropriate to be as direct as the wording used in the examples on the left.

The following examples show how this technique can either reinforce the importance of a recommendation (closer) or provide distance from a potentially worrisome symptom. The counselor's judgment determines which is most appropriate.

\section{Closer}

Kendra should have periodic examinations of her skin to check for neurofibromas.

Since Bob has Marfan syndrome, he is at a significantly increased risk for aortic dissection and should have a yearly echocardiogram.

\section{More distant}

Children with neurofibromatosis (NF) should be checked for changes in their skin, such as neurofibromas. Individuals with Marfan syndrome should have yearly echocardiograms.

Another tool for conveying distance is to eliminate mentioning of an individual. Obviously the letter may be discussing a patient, but the patient need not be directly referred to in every sentence. In passages that present information that is understood to be troubling, the patient can be left out entirely as illustrated in the following examples:

\section{Include patient}

Nisha is likely to lose her hearing.

You have a $50 \%$ chance for having a child with neurofibromatosis.

\section{Exclude patient}

Children with this condition tend to lose their hearing.

An individual with neurofibromatosis has a $50 \%$ chance for passing the gene that causes NF on to his or her children.

\section{Distance: Active Voice vs. Passive Voice}

Active and passive voice are rhetorical devices that can be used to influence distance in patient letters. Their application can be a very effective means of shaping and emphasizing points in the patient letter. If not already a familiar tool, practice in the use of active and passive voice will be of great benefit to those developing letter-writing skills. In general writing and speech, the active voice is much preferred over the passive voice (Blake and Bly, 1991; Venolia, 1982). However, our field's grounding in the basic science literature, which generally uses the passive voice, and our foundation in nondirective counseling can make it challenging to use the active voice in professional writing. Situations in 
which the passive voice may be preferred over the active voice include instances in which a person taking an action is either unknown or less important than the action itself (Blake and Bly, 1991). In patient letters one can alternate between active and passive voice. However, readers generally prefer the active voice, and it should be used as much as possible unless one is trying to convey more distance through use of the passive voice (Tarshis, 1992).

The active voice indicates that the subject is acting or doing. The passive voice indicates that the subject is being acted upon or is having something done to it (Tarshis, 1992).

\section{Active}

Christina is experiencing increased nystagmus (jerky eye movements).

(The subject is Christina. She is doing something, is experiencing.

You decided to proceed with genetic testing for cystic fibrosis.

(The subject is you, the patient. You have done something, decided to proceed.
Passive

Nystagmus was noted to be more pronounced during Christina's exam.

(The subject is nystagmus. It was noted to have changed, to be more pronounced.

The decision was made to proceed with genetic testing for cystic fibrosis.

(The subject is the decision that has been made).

The active voice tends to come across more strongly and more vividly than the passive voice. The subject in the active voice is more responsible for and closer to the action in the sentence, since the subject is performing the action. The subject in the passive voice is less responsible for and more distant from the action, since the action is being performed by a separate force upon the subject.

\section{Distance: Point of View}

While most formal writing is written entirely in the third person, patient letters usually refer to the second and first person as well. All three can be used interchangeably throughout the letter. When writing or speaking one can refer to the first, second, or third person, according to how close or distant one wants either the genetics team or the patient to be in relation to the information.

First Person: I, We

Second Person: You

Third Person: He, She, It

Information expressed in the first person is not at all distant from the speaker and therefore carries the most influence. In the first person one is speaking for oneself (the counselor, the staff: "I, we"). 
Examples: I recommend that ..., We referred you to ...,

The first person voice seems natural when reviewing recommendations or referrals made during the visit or when commenting on more personal/individual aspects of the visit.

Examples:

We recommend that an annual thyroid screen be performed on Joseph by his pediatrician.

We appreciate all your efforts to provide a complete family history.

The following example illustrates the difference in weight that third and first person convey:

\section{Third person}

A referral to ophthalmology for evaluation is indicated.

\section{First person}

We referred you to an ophthalmologist for evaluation.

One does not write in the second person as one writes in the first and third person, but one can address the second person. Addressing the second person (the patient: you) is direct, personal and obviously closer to the patient than the third person:

Example: You and your children have all undergone testing for ...

This is often used when describing or reporting family, medical, developmental, or pregnancy history that the patients themselves provide.

Examples: You reported ..., stated ..., described ..., indicated ...

The most distant point of view is the third person in which no specific personal references is made, either to the speaker (the counselor or staff) or to the person being spoken to (the patient). Rather, reference is made to a third party or thing. Using the third person, it is possible to make no specific references to any individual at all (although those references can generally be inferred from surrounding context in the letter).

\section{Examples:}

The radiology report indicated...

The physical examination showed...

Children with XYZ syndrome should be monitored for ...

The clinical features of neurofibromatosis include...

The third person conveys a strong sense of distance and generality. It is easy to write the majority of the patient letter (e.g., review or summation of relevant information regarding the clinic visit) in the third person. 


\section{Distance: Definitive vs. Nondefinitive Statements}

Another device, which influences distance, is the definitiveness of statements. Counselors have the choice with each statement they make to be very specific (definitive) or noncommittal (nondefinitive). Issues counselors are absolutely certain about can be stated definitively; information involving personal judgment, uncertainty, or less objectivity should be worded as nondefinitive statements.

\section{More definitive}

Your radiographs showed ...

Paul does not have Marfan syndrome.

\section{Less definitive}

Your radiographs were suggestive of ... Available information indicates that Paul probably does not have Marfan syndrome.

\section{Use of Value-Free and People-First Language}

Language in the patient letter can be kept relatively value-free by using neutral, nonstigmatizing terms and by avoiding the use of slang or words with a strong negative meaning. One approach used by genetic counselors is to distinguish the description of a tissue, organ system, or protein as abnormal, deficient, defective, incomplete, etc., but to avoid using these connotative terms to describe people.

\section{Examples:}

Marfan syndrome is due to an abnormality of connective tissue.

A person who inherits one copy of the gene mutation (altered gene) is referred to as a carrier.

A child who inherits one copy of the gene mutation (nonworking gene) will be affected with the condition.

\section{Stigmatizing}

Your daughter is afflicted with Down syndrome.

As an unwed mother, you described the challenges of caring for a child with Duchenne Muscular Dystrophy (DMD).

\section{Value-free}

Your daughter has Down syndrome.

As the sole provider for your child, you described the challenges of caring for a child with DMD.

People-first language employs the use of identifiers prior to the modifier that is being used to describe the person. 
Modifier-first

Your Down syndrome baby...

A blind child will benefit from...

Your mentally retarded cousin...
People-first

Your baby with Down syndrome

As a child with blindness, Maria will benefit from...

Your cousin, who has mental retardation, ...

\section{Presentation of Patient Affect/Response/State of Mind}

The counselor should take care to report things from his own point of view and not to speak for others. The counselor cannot accurately write that the patient was in a particular state of mind, but can write that he perceived the patient as being in a particular state of mind. Likewise, the counselor should be careful not to attribute patient behavior definitively to specific patient motives as illustrated in the following examples:

\section{Unsubstantiated}

You decided not to become pregnant because of your risk for...

You were upset by the news of the diagnosis.

You are anxious about having another child with the same condition.

\section{More accurate}

You indicated that one of the reasons for your decision not to become pregnant was your risk for...

You seemed upset by the news of the diagnosis.

You expressed concern about having another child with the same condition.

\section{CONCLUSION}

These guidelines were developed to help illuminate some of the characteristics of patient letters and to promote their consideration by students of genetic counseling and by the professional community. These guidelines are intended as a starting point for teaching the skill of patient letter-writing and will benefit from modification by individuals, training programs, and clinical centers in accordance with their own specific methods, criteria, and personal styles. It is important that our understanding of the methods used in writing letters continue to be examined and clarified.

\section{APPENDIX}

\section{Sample Patient Letter Illustrating Content and Wording}

This sample letter illustrates several components of patient letters. The comments section, at the left, discusses the application of elements from the guidelines. 
Date

RE:

\section{REG\#}

The Introductory paragraph includes visit date, clinic name, reason for visit, and purpose of letter.

The letter is lacking complete historical information, although some is included in the introductory paragraph. One may wish to include more complete historical information in a separate paragraph following the introduction.

Suggestions:

${ }^{1}$ Define these terms in everyday language.
${ }^{2}$ This is an example of the use of distance that may modulate the impact of powerful news.
Dear Mr. and Mrs.

We are sending this letter to summarize our meeting on (date), in the Pediatric Genetics Clinic at the University of Medical Center. Your son, Philip, was referred by Dr. of the Division of Pediatric Orthopedics for evaluation for possible neurofibromatosis. As you are aware, Philip was diagnosed as having a pseudoarthrosis ${ }^{1}$ of the left tibia ${ }^{1}$ and also was noted to have several cafe-au-lait spots ${ }^{1}$ both of which are features of neurofibromatosis.

Neurofibromatosis $1(\mathrm{NF})$ is a progressive disorder of the skin and nervous system that occurs in about 1 in every 3000 individuals. The features of NF include: cafe-au-lait spots, axillary and inguinal ${ }^{1}$ freckling, neurofibromas (small tumor-like growths), Lisch nodules (areas of pigment in the iris), congenital defects of the bones, optic gliomas (a tumor of the optic nerve), and a first degree ${ }^{1}$ relative with NF. It is a highly variable condition involving only cafe-au-lait spots in some individuals to numerous neurofibromas and other more serious findings in others. Approximately $80 \%$ of individuals affected with NF have mild to moderate symptoms; about $20 \%$ have more significant problems. It is not possible to predict in advance which individuals will develop the more serious complications of NF.

In order to make the diagnosis of NF, at least two of the seven features described above must be present. Philip has multiple cafe-au-lait spots, inguinal freckling, and a pseudoarthrosis. Therefore, he has the necessary criteria for making the diagnosis of $\mathrm{NF}^{2}$ It is not possible to know whether he will develop symptoms over time consistent with mild to moderate NF or 


\footnotetext{
${ }^{3}$ This is a statement about which the counselor cannot be certain.

${ }^{4}$ This is an example of excluding mention of staff, which increases distance. Alternatively, using first person reference to the staff would decrease distance, thereby strengthen the recommendation.
}

${ }^{5}$ Use of the word condition rather than disorder is more value-free.

${ }^{6}$ The information about chromosomes is not necessary for explaining the genetics of NF and could be eliminated.

${ }^{7}$ This is an example of language that is not value-free nor people-first. whether he will have more serious complications. However, as indicated above, he will, more than likely ${ }^{3}$ have only mild to moderate symptoms. At the present time, it is recommended that Philip continue having ${ }^{4}$ yearly pediatric examinations and in addition, a yearly evaluation by ophthalmology (including formal assessment of peripheral vision) and genetics (genetics visits could alternate with pediatrics so that he is seen at 6 month intervals). Children with NF should be monitored for height, weight, head circumference, blood pressure, vision and hearing, evidence of scoliosis, normal sexual development, and any changes in the skin such as neurofibromas. Of course, in addition, Philip needs continued follow-up with orthopedics.

Neurofibromatosis is inherited as an autosomal dominant condition ${ }^{5}$. It results from the presence of a single abnormal gene. By way of review, genes are the basic units of hereditary information. Genes code for all the body's traits and functions. Genes occur in pairs, so that there are two matching genes that code for each trait. Genes are carried on larger structures known as chromosomes. Chromosomes also occur in paris; one member of each chromosome pair (and thus one member of each gene pair) is from one parent; the other is from the other parent. ${ }^{6}$ Since one member of every gene pair is present in each egg or sperm, an affected $^{7}$ individual has a $50 \%$ chance for transmitting the $\mathrm{NF}$ gene to his or her children, who then may have similar, milder, or more severe symptoms.

Approximately one-half of individuals who have NF have inherited the NF gene from one of their parents. The other half do not have an apparently affected parent; the NF gene in these individuals arose as a new mutation (a spontaneous gene change) in either the egg or sperm cell of one of the parents. Careful physical examinations of each of you are therefore recommended to check for symptoms and signs of NF in order to determine whether Philip inherited the NF gene from one of you or whether it arose for the first time in him. 
${ }^{8}$ This paragraph and paragraph \#3 both contain recommendations, which could be combined in just one location.
This has importance in terms of your own medical care, and it has implications for other family members. If one of you is found to have NF, then the risk for recurrence of NF in any future pregnancy is $50 \%$. If neither of you is found to have NF, then the risk for recurrence is probably not significantly greater than the general population risk of less than $1 \%$.

Several specific recommendations were made at the time of our meeting ${ }^{8}$. It is recommended that Philip have a MRI of the brain because of his history of speech delay and in view of his diagnosis of NF. In addition, a consultation with Physical Medicine \& Rehabilitation is recommended to determine whether an exercise program is indicated because of the need for bracing of his left leg.

Additional information in the form of written materials prepared by the National Neurofibromatosis Foundation, Inc. were provided to you during your visit. The local chapter of the NF Foundation may be an additional source of materials and support. Their phone number is Should there be any additional questions or concerns regarding our meeting, please feel free to call our office at the number above. Otherwise, we look forward to meeting with you again at the time of your follow-up appointment.

Sincerely,

Genetic Counselor

Division of Pediatric Genetics

Division of Pediatric Genetics

Assistant Professor of Pediatrics and Human Genetics

cc: Dr.

Orthopedics

Dr. 


\section{ACKNOWLEDGMENTS}

This work has been supported by the National Society of Genetic Counselors (Special Project Award, 1996) and by the University of Michigan, Departments of Human Genetics, Internal Medicine and Pediatrics. The authors wish to thank the program directors, supervisors, students, and practicing genetic counselors who contributed to the development of these guidelines.

\section{REFERENCES}

Blake G, Bly RW (1991) The Elements of Business Writing. New York: Macmillan.

Hallowell N, Murton F (1998) The value of written summaries of genetic consultations. Patient Educ Couns 35:27-34.

Kelly PT (1977) Dealing With Dilemma: A Manual for Genetic Counselors. New York: Springer.

National Society of Genetic Counselors (1992) Code of ethics of the National Society of Genetic Counselors. J Genet Couns 1:41-43.

Strunk W, White EB (1979) The Elements of Style, 3rd edn. New York: Macmillan.

Tarshis B (1992) Grammar for Smart People. New York: Pocket Books.

Venolia J (1982) Better Letters. Berkeley: Ten Speed Press. 